\title{
All-Fiber Interferometer for Chromatic Dispersion Measurements
}

\author{
LUC THEVENAZ, JEAN-PAUL PELLAUX, AND JEAN-PIERRE VON DER WEID
}

\begin{abstract}
A new all-fiber interferometric method for chromatic dispersion measurements in meter-length single-mode fibers is presented. In a Michelson setup a reference fiber is used whose physical length is varied so as to obtain an adjustable optical delay. Time resolution, ease of manipulation, and mechanical isolation are considerably improved with respect to conventional interferometers. Resolution of group delay measurement and chromatic dispersion over the full $1100-1700 \mathrm{~nm}$ spectral range are better than 5 fs and $0.1 \mathrm{ps} / \mathrm{nm} \cdot \mathrm{km}$, respectively.
\end{abstract}

\section{INTRODUCTION}

$S^{\prime}$ NGLE-MODE optical fibers are of great interest for high-data-rate long-span transmission systems because of their very low attenuation and high bandwidth at wavelengths from 1.3 to $1.55 \mu \mathrm{m}$. However, chromatic dispersion of the fiber, together with the finite spectral width of the light source, give rise to pulse broadening which limits the overall bandwidth attainable with such systems. Measurement of chromatic dispersion is then of utmost importance for users and manufacturers of singlemode fibers in order to insure optimal performance.

Low dispersion $(<5 \mathrm{ps} / \mathrm{nm} \cdot \mathrm{km})$ fibers are now commonly available in the zero material dispersion window $(1.3 \mu \mathrm{m})$ and more recently in the minimum attenuation window $(1.55 \mu \mathrm{m})$-the so-called dispersionshifted fibers. These low dispersion values require very accurate measuring systems. Several experimental methods have been proposed during the last few years, most of them measuring the group delay as a function of wavelength and obtaining chromatic dispersion by simple numerical differentiation.

Fiber Raman lasers [1] are now commonly used as pulsed light sources for the measurement of the group delay at the Raman shifted wavelengths. Comprehensive results are directly obtained, but an expensive setup and safety care are required. Furthermore it is difficult to get a resolution better than $50 \mathrm{ps}$ on the group delay measurement. Another proposal is to sinusoidally modulate an LED [2] whose spectrum is scanned by means of a monochromator and to measure the group delay by detecting the phase of the modulation signal at each wavelength. Resolution is theoretically good, but is strongly

Manuscript received August 22, 1986. This work was supported by Swiss National Fund for Scientific Research and by the Brazilian National Council for Scientific and Technological Development (CNPq).

L. Thévenaz and J.-P. Von der Weid are with the University of Geneva, Group of Applied Physics, 1211 Geneva 4, Switzerland.

J.-P. Pellaux is with Alphatronix, 1228 Geneva, Switzerland.

IEEE Log Number 8717054. reduced by the low detected intensity and therefore the poor signal-to-noise ratio. In addition several LED's are needed to cover the full spectral range of interest. Signalto-noise ratio can be increased by replacing the LED and monochromator by an array of semiconductor lasers, each of them emitting at a different wavelength. However, many lasers are necessary for a good resolution and a wide spectral range, rapidly raising the cost to prohibitive levels.

Interferometric measurements with coherence limited sources had been reported by many authors. Some of them use LED or lasers [3]-[5], others a simple halogen lamp [6]. The stability of the interferometer limits the actual length of the measured samples to roughly $1-5 \mathrm{~m}$ but reported resolution of $0.1 \mathrm{ps} \mathrm{[6]-[7]} \mathrm{indicates} \mathrm{that} \mathrm{this}$ method can be very accurate. In addition the method seems attractive because of its low cost and theoretically unlimited spectral range. It seems, however, to have retained little attention, probably because of the expected difficulty of getting interference fringes in such a longarmed interferometer.

In this paper we report a novel interferometric technique for the determination of chromatic dispersion. It allows the measurement of the group delay with a resolution better than $5 \mathrm{fs}$ which is at least one order of magnitude better than previously reported results. Our method has a broad wavelength coverage from 1.1 to $1.7 \mu \mathrm{m}$ and can be used for fiber samples lengths of 1-2 m. It is based on a Michelson configuration in which a reference fiber is placed in one arm of the interferometer and whose length is elastically varied resulting in a precisely adjustable optical delay length. The setup is simple to make, easy to manipulate, and inexpensive. Neither IR visualizing camera nor lasers are needed.

\section{Basic Principles}

Most of the experimental methods for chromatic dispersion measurement are based on the direct measure of the group delay $\tau$ as a function of wavelength, the chromatic dispersion being obtained by simple differentiation of $\tau(\lambda)$ :

$$
D(\lambda)=\frac{1}{L} \frac{d \tau}{d \lambda}
$$

where $L$ is the length of the light path in the dispersive medium.

The interferometric method for measuring the group de- 
lay is based on the fact that when coherence limited light is splitted and recombined in a two arms interferometer, interference fringes can be detected only if the group delay in both arms are nearly equal. The intensity at the recombination point is given by

$$
\left\langle\left|E_{1}+E_{2}\right|^{2}\right\rangle=\left\langle\left|E_{1}\right|^{2}\right\rangle+\left\langle\left|E_{2}\right|^{2}\right\rangle+2 \operatorname{Re}\left\langle E_{1}^{*} E_{2}\right\rangle
$$

where $E_{1}$ and $E_{2}$ are the field amplitudes of the light coming from each arm of the interferometer and the brackets mean time average.

The third term in the right hand side of (2) is the crosscorrelation of the field amplitudes which depend on the source spectrum and the dispersive properties of the media in each arm. Writing down the field amplitude $E_{i}$ as

$$
\begin{gathered}
E_{i}=\int d \omega^{\prime} F\left(\omega^{\prime}\right) \exp \left[-i \beta_{i}\left(\omega^{\prime}\right) z_{i}\right] \cdot \exp \left(i \omega^{\prime} t\right) \\
i=1,2
\end{gathered}
$$

where $F(\omega)$ is the amplitude spectrum of the light source, $\beta_{i}(\omega)$ the propagation constants along each arm $i$, and $z_{i}$ the corresponding length, the cross-correlation term gives

$$
\begin{aligned}
\left\langle E_{\uparrow}^{*} E_{2}\right\rangle= & \int d \omega^{\prime}\left|F\left(\omega^{\prime}\right)\right|^{2} \\
& \cdot \exp \left\{i\left[\beta_{1}\left(\omega^{\prime}\right) z_{1}-\beta_{2}\left(\omega^{\prime}\right) z_{2}\right]\right\} .
\end{aligned}
$$

For nearly monochromatic light of frequency $\omega,|F(\omega)|^{2}$ is nonzero only in a limited range $\delta \omega$ around $\omega$, whereas $\beta_{i}(\omega)$ are slowly varying functions of $\omega$. Taking the Taylor's expansion series of $\beta_{i}$ and keeping only the first order terms:

$$
\beta_{i}\left(\omega^{\prime}\right)=\beta_{i}(\omega)+\frac{d \beta_{i}}{d \omega}\left(\omega^{\prime}-\omega\right)+\cdots
$$

equation (4) now reads:

$$
\begin{aligned}
\left\langle E_{1}^{*} E_{2}\right\rangle= & e^{i \Phi} \int d \omega^{\prime}\left|F\left(\omega^{\prime}\right)\right|^{2} \\
& \cdot \exp \left\{i\left[\tau_{1}(\omega)-\tau_{2}(\omega)\right]\left(-\omega^{\prime}\right)\right\}
\end{aligned}
$$

where $\tau_{i}(\omega)=\left(d \beta_{i} / d \omega\right) z_{i}$ are the group delays in each arm of the interferometer and $\Phi$ is a phase factor given by

$$
\Phi=\beta_{1}(\omega) z_{1}-\beta_{2}(\omega) z_{2} .
$$

When the length of one arm, say $z_{1}$, is continuously varied the phase factor $\Phi$ gives rise to the oscillatory behavior of the light intensity which characterizes the interference fringes. The amplitude of the intensity oscillations is the Fourier transform of the power spectrum of the source centered at the position where the group delays in both arms are equal. This means that once the group delay in the reference arm is known, the group delay of the second arm can be measured by scanning the reference group delay and searching for the center of the resulting fringe pattern. The effect of the neglected second-order term in the Taylor's expansion (5) is to broaden the interference

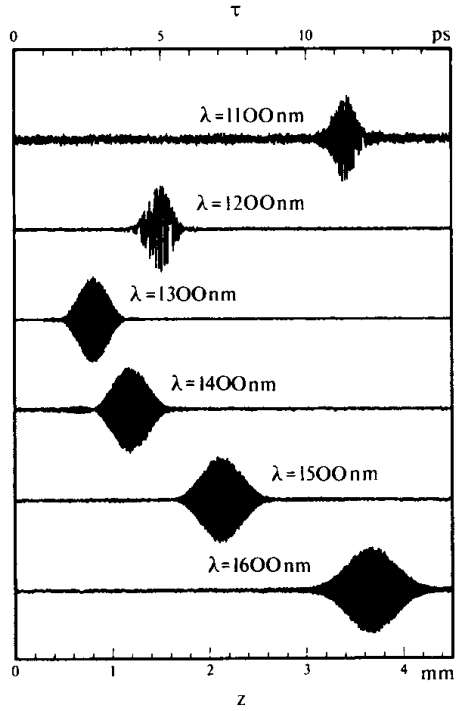

Fig. 1. Interference patterns at different wavelengths produced by a Michelson interferometer with a $1.8-\mathrm{m}$ single-mode fiber balanced by a 2.7-m air path.

pattern and to reduce fringes contrast. Some authors used this property for a direct measure of chromatic dispersion [8], [9]. They performed measurements either in the highly dispersive range around $800 \mathrm{~nm} \mathrm{[8]}$ or with a wide spectrum source at $1300 \mathrm{~nm}$ [9].

Delay scanning is usually obtained by introducing a variable air path in the reference arm using a roof prism or a system of mirrors on a displacement unit, so that the length of a parallel beam in air is varied. Shifts of the fringe pattern for different wavelengths can be seen in Fig. 1 for an interferometer with a $\sim 2.7$-m-long variable air path balanced by a single-mode fiber. In this case the center of the fringe pattern $z(\lambda)$ is directly proportional to the group delay of the single-mode fiber $\tau(\lambda)=z(\lambda) / c$.

Another possibility consists in introducting a length $l_{0}$ of a fiber whose group index $N_{0}(\lambda)$ is known in the reference arm of the interferometer, in addition to a length $\delta z$ of variable air path. In this case, the center of the fringe pattern will be given by the difference between the unknown fiber and the reference fiber

$$
\tau(\lambda)=\frac{N_{0}(\lambda) z_{0}}{c}+\frac{\delta z}{c} .
$$

In our work, a novel interferometric configuration has been investigated in which the variable air path is suppressed and delay scanning is obtained by elastically changing the length of the reference fiber in an all-fiber interferometer. It is well-known that elongations up to 2 percent of the original fiber length can be easily obtained without damage in optical fibers. However, since optical properties of the fiber change with stress, refractive index changes must be taken into account in order to convert length measurements into time delays. Let $N_{0}(\lambda)$ and $z_{0}$ be the group index and length of the reference fiber in 


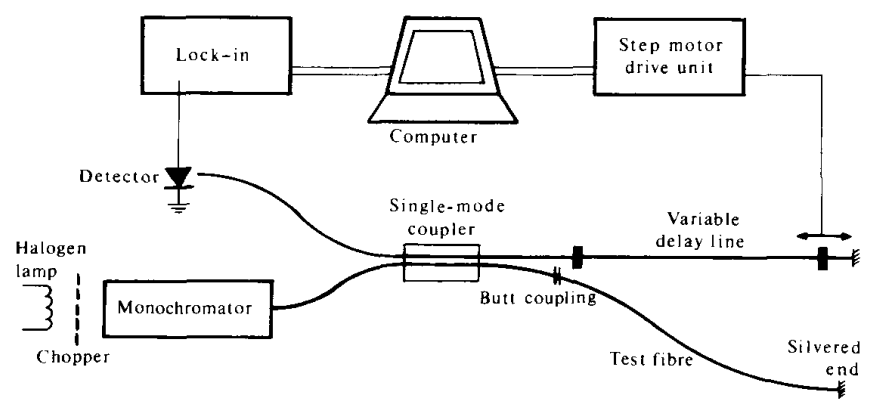

Fig. 2. All-fiber setup for interferometric measurements of single-mode fibers in the $1100-1700 \mathrm{~nm}$ range.

unstressed conditions. Up to the first order the group delay may now be written as

$$
\tau(\lambda)=\left[N_{0}(\lambda)+\frac{d N}{d z} \delta z\right] \frac{z}{c}
$$

where $z$ is the actual length of the reference fiber at the center of the fringe pattern and $\delta z=z-z_{0}$ its elongation. Keeping the first-order approximation, the group index variation $\delta N / N_{0}$ is related to the induced strain $\delta z / z_{0}$ by [10]:

$$
\frac{\delta N}{N_{0}}=-\frac{N_{0}^{2}}{2}\left[p_{12}-\nu\left(p_{11}+p_{12}\right)\right] \frac{\delta z}{z_{0}}
$$

where $p_{i j}$ are elements of the strain-optic tensor and $\nu$ is the Poisson's ratio. Replacing (10) into (9) and keeping aside second-order terms in $\delta z$ one obtains

$$
\tau(\lambda)=\frac{N_{0}(\lambda) z_{0}}{c}+\Gamma \frac{\delta z}{c}
$$

where

$$
\Gamma=N_{0}\left\{1-\frac{N_{0}^{2}}{2}\left[p_{12}-\nu\left(p_{11}+p_{12}\right)\right]\right\} .
$$

The first term in the right-hand side of (11) plays the role of a known fiber of length $l_{0}$ in the reference arm, whereas the second term plays the role of variable delay. Although the $\Gamma$ factor in (11) contains implicitly a $\lambda$-dependence through the group index $N_{0}(\lambda)$ its variation in the region of minimum dispersion is expected to be very small so that to within $\sim 0.1$ percent $\Gamma$ is expected to be independent of wavelength unless $p_{i j}$ vary with $\lambda$. Using the parameters available in the literature for bulk silica [11] $\Gamma$ is estimated to be 1.14 . However, discrepancies between experimental and theoretical calculations of elastooptic properties in optical fibers had been reported [12] so that a careful calibration of $\Gamma$ must be done in order to check its value and its $\lambda$-dependence.

\section{AlL-FIBER INTERFEROMETER}

The all-fiber interferometer used in our experiment is outlined in Fig. 2. The light source was a halogen lamp filtered by a $20-\mathrm{cm}$ grating monochromator and the detec tor was a pig-tailed InGaAs p-i-n photodiode. A single- mode coupler was used instead of the classical beamsplitter in a Michelson configuration. Coherent coupling insures ideal conditions for maximum fringes contrast without the need of achromatic optics, careful mirror alignment, and IR viewing equipment. However, singlemode couplers usually have a wavelength dependent coupling ratio. Good fringes contrast is obtained if the coupling ratio is bounded between 20 and 80 percent which actually defines the spectral range covered by the interferometer. The stretched fiber used as variable path together with the single-mode coupler completely eliminates the need for achromatic injection optics and parallel beam reconstruction. The mechanical stability of the allfiber interferometer was therefore greatly enhanced with respect to conventional systems.

The Michelson configuration was chosen rather than the Mach-Zehnder previously reported in interferometric measurements [3]-[6] because the light travels twice the length of the fibers and hence the dispersion effects are doubled. The reflection at the fiber endfaces is obtained by a chemical deposition of silver at the cleaved endfaces. No additional alignment of mirrors at the fibers endfaces is needed but good quality cleaved endfaces are required for best fringes contrast.

Alignment of the test fiber is obtained by searching for the signal reflected at the silvered fiber end. For best signal-to-noise ratio the halogen source is chopped and the reflected signal is lock-in amplified.

Index matching oil insures optimum transmission at the single-mode fiber-to-fiber butt coupling also eliminating undesirable Fresnel reflections at the interfaces.

The reference arm of the all-fiber Michelson interferometer contains a 1.5 -m-long primary coated single-mode fiber anchored at a fixed support at one end and at a 0.1 $\mu \mathrm{m}$ sensitivity stepping motor driven displacement unit at the other end. Primary coating was removed at the anchoring points to prevent the stretched fiber from sliding on its supports. In order to avoid fatigue effects that could reduce the lifetime of the stretched fiber we restricted the strain to a maximum of 0.5 percent. Sweeping the length of the reference arm allows direct observation of the interference fringes at the output of the lock-in amplifier as shown in Fig. 1. This direct measurement needs, however, a slow sweeping speed imposed by the integration time of the lock-in amplifier. 


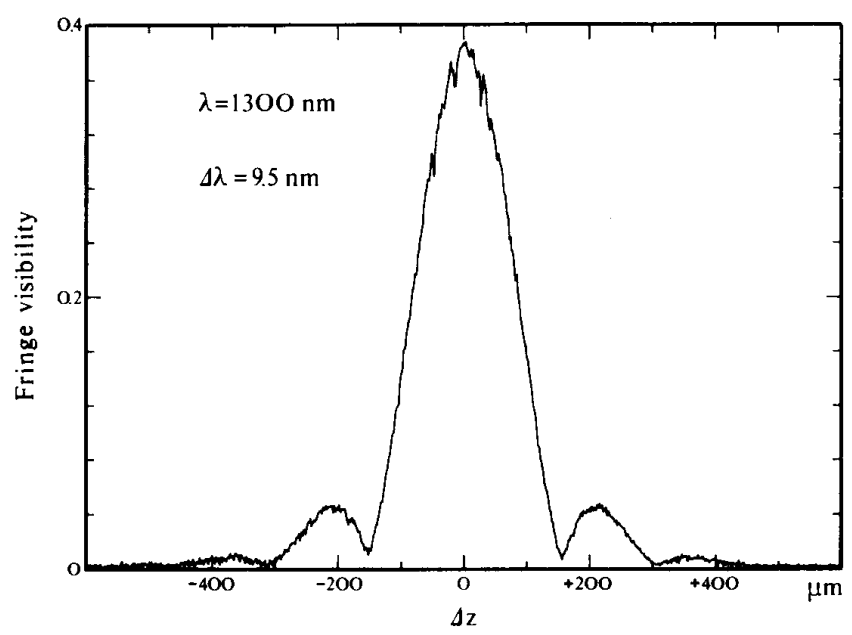

Fig. 3. Direct measurement of the fringe visibility with a tuned amplifier.

On the other hand sweeping the length of the reference arm at constant rate gives rise to an oscillatory signal whose amplitude can be directly measured with a tuned amplifier at the correct frequency $f$ :

$$
f=\frac{2 \Gamma v}{\lambda}
$$

where $v$ is the sweeping speed, $\lambda$ is the wavelength of the measuring light, and $\Gamma$ is the factor defined in (12). Therefore the fringe visibility is directly measured as shown in Fig. 3 and a much better accuracy is obtained in the computation of the center of the pattern. Here the two-phase lock-in amplifier shown in Fig. 2 was used as tuned amplifier, its internal oscillator being locked at the frequency given by (13). With this method the measurement time for each experimental point falls from $\sim 10$ min down to $\sim 15 \mathrm{~s}$.

Calibration of the $\Gamma$ factor defined in (12) was done by replacing the test fiber in Fig. 2 by a variable air path whose length at the center of the fringe pattern is a direct measurement of $\tau(\lambda)$. The slope of the linear relationship between $\tau(\lambda)$ and the elongation $\delta z$ is therefore a direct measurement of the $\Gamma$ factor. This is the most accurate optical path measurement which can be done in singlemode optical fibers. The mirror at the end of the variable air path was mounted on a $0.1-\mu \mathrm{m}$ stepping motor driven displacement unit so that both elongation and optical path change could be measured with the same sensitivity. Calibration curves were made in the $1100-1700 \mathrm{~nm}$ range for several different step-index fibers. Results for the two extreme index differences are plotted in Fig. 4 and clearly state that the $\Gamma$ factor remains constant over the whole $1100-1700 \mathrm{~nm}$ range. No hysteresis or sliding effects were observed even after a great number of stretching cycles confirming the good quality of the anchorages of the stressed fiber. Assuming $\Gamma$ to be wavelength independent and taking the scattering of the data in Fig. 4 as experimental errors we determined the value of the $\Gamma$ factor as
$1.171 \pm 0.002$. The same result was obtained for a fiber with coating removed so that it suggests that the measured $\Gamma$ factor is intrinsic to the fiber material and not affected by the coating.

Finally, calibration of the dispersion of the reference fiber was done with the same configuration described for the measure of the $\Gamma$ factor. The nondispersive air path was used as absolute reference medium and the length of the stretched fiber was swept at constant speed in order to detect the center of the fringe pattern at each wavelength. An average over several dispersion measurements gave an extremely accurate calibration curve which was permanently stored onto the computer storage medium and subtracted from the delay measurements of each test fiber following (11).

In addition to the calibration curve, the dispersion of the reference fiber was measured under stress in order to verify if the change in waveguide parameters, like core radius and index difference, could affect the dispersion properties of the reference fiber. No measurable effect was observed for strains up to 2 percent on fibers with low waveguide dispersion. Nevertheless a fiber with strong waveguide dispersion like a dispersion-shifted fiber should not be used as a variable delay line because the dispersion could be altered enough to influence the measurements.

\section{Results AND Discussion}

Measurements of group delay for a conventional stepindex and a dispersion shifted fibers are shown in Fig. 5(a) with 1.62-m samples length. The measured group delays were fit to a fourth degree polynomial and the corresponding computed chromatic dispersions are shown in Fig. 5(b).

The accuracy in the determination of the center of the fringe pattern depends on the coherence length $l_{c}$ of the source which is related to its spectral width $\delta \lambda$ by

$$
l_{c}=\lambda^{2} / \delta \lambda \text {. }
$$




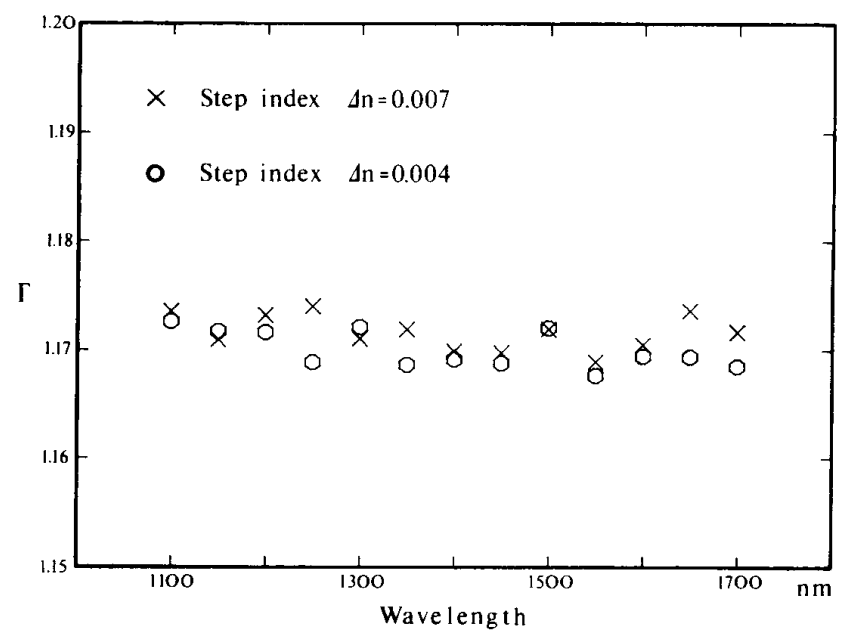

Fig. 4. $\Gamma$ factor measured at different wavelengths for two different stepindex fibers.

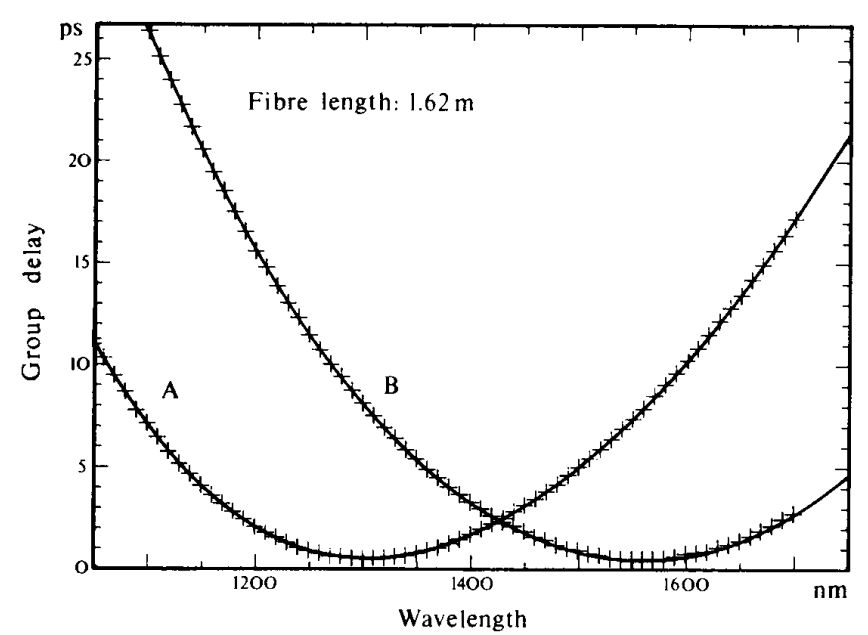

(a)

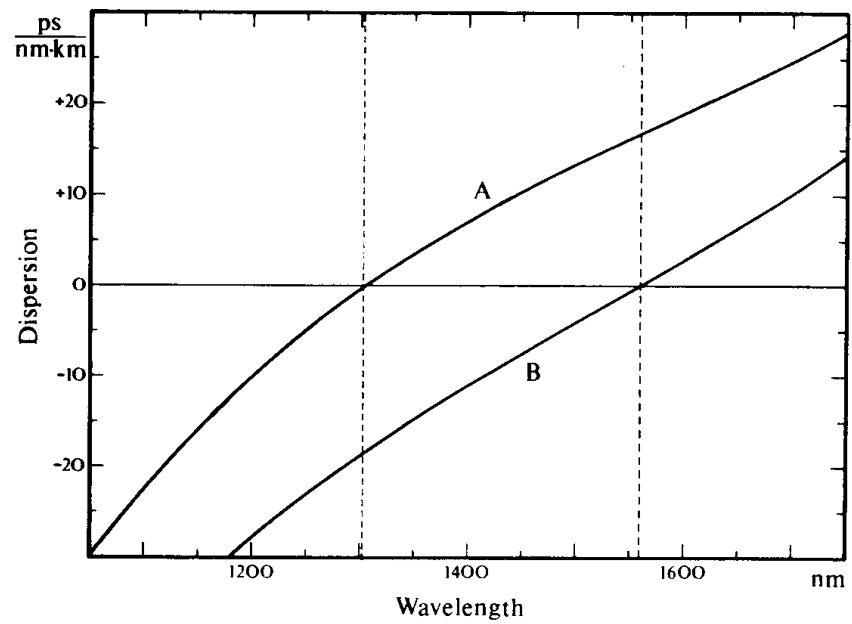

(b)

Fig. 5. (a) Interferometric measurements of group delay spectra. $A$ : stepindex fiber. $B$ : dispersion-shifted fiber. (b) Computed dispersion spectra. Zero-dispersion wavelengths: $A=1302 \mathrm{~nm}, B=1559 \mathrm{~nm}$. 


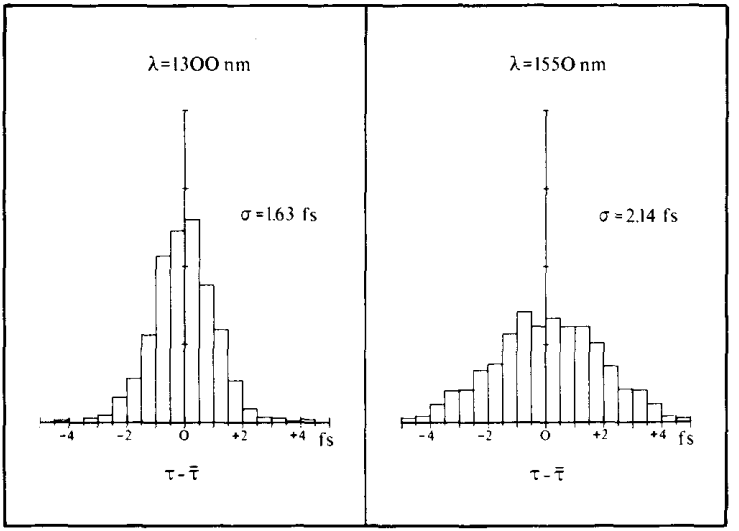

Fig. 6. Experimental deviations on group delay measure computed with 1000 measurements sample at two different wavelengths. Vertical scale is arbitrary.

A too narrow spectral width gives rise to a broad fringe pattern whose center will be known with little accuracy. On the other hand a broad spectral width will give a sharp fringe pattern but the wavelength accuracy will be correspondingly reduced. Interferometric measurement of the group delay is actually limited by the uncertainty principle which states the incompatibility between time and energy measurements. Best results were obtained with a $\sim$ 10-nm spectral width source at $1300 \mathrm{~nm}$, but results at $1550 \mathrm{~nm}$ are slightly less accurate due to the greater coherence length with increasing wavelength.

Experimental accuracy was investigated by repeating the same group delay measurement maxiy times. Results are outlined in Fig. 6 for 1300 and $1550 \mathrm{~nm}$. The measured $\sim 2$-fs standard deviation is more than one order of magnitude better than previously reported accuracies [7]. This very high precision is mainly due to the direct detection of the fringe visibility, the numerical treatment of the signal, and the algorithm used for the calculation of the center of the fringes pattern. In addition, the all-fiber interferometer is much less sensitive to mechanical perturbation than conventional air path interferometers.

The wavelength of zero dispersion of a test fiber was measured many times giving a reproducibility better than $1 \mathrm{~nm}$ for a zero dispersion near $1300 \mathrm{~nm}$ and better than $2 \mathrm{~nm}$ for dispersion shifted fibers at $1550 \mathrm{~nm}$. This difference is mainly due to the slightly less accurate delay measurements at $1550 \mathrm{~nm}$.

Since the dispersion curve is calculated from the fit of the time delay experimental data, the wavelength of zero dispersion $\lambda_{0}$ is slightly dependent on the kind of theoretical fitted curve. Interferometric measurements are a very powerful tool for this approach as it allows an arbitrary number of experimental points in the delay curve. For delay measurements each $10 \mathrm{~nm}$ over the whole 1100 $1700 \mathrm{~nm}$ spectral range a fourth degree polynomial fit and a five terms Sellmeier's expansion gave equivalent results fór both standard and dispersion-shifted fibers. Typical $\chi^{2}$ standard deviations between experimental data and the fit- ted theoretical curves are 20 fs for a standard step-index fiber and $30 \mathrm{fs}$ for a dispersion-shifted fiber. The description of the delay data by a three terms Sellmeier's expansion over the full $1100-1700 \mathrm{~nm}$ spectral range gave less accurate results, the standard deviation between the calculated and measured delay data increasing to $40 \mathrm{fs}$ for a standard fiber and up to 200 fs for a dispersion-shifted fiber. This result clearly indicates the inadequacy of the three terms Sellmeier's expansion in the description of group delay data over a wide spectral range.

On the other hand adding more terms to the polynomial or Sellmeier's expansion fit did not improve the quality of the theoretical fit, so that the best results were obtained with the fourth degree polynomial or the five terms Sellmeier's expansion. It is interesting to note that standard deviation between the calculated and measured delay data is somewhat larger than the experimental accuracy related to the delay measurements. This difference is due to regular systematic deviations between the experimental data and the fitted curve. These deviations depend on the fiber being measured and change with bending applied to the fiber, suggesting they are probably related to polarization effects due to residual birefringence. Further experiments are under way in order to clarify this point.

\section{CONCLUSION}

In summary, a novel experimental setup based on a Michelson interferometric method was developed for chromatic dispersion measurements of 1-2 m optical fiber samples. In one arm of the interferometer a reference single-mode fiber is placed whose length is varied so as to obtain an adjustable and highly reproducible delay, the other arm being the fiber sample to be measured. The allfiber configuration replacing the classical beamsplitter by a single-mode coupler and the variable air path by the stretched reference fiber allows a resolution on time delay measurements of $5 \mathrm{fs}$. This is more than one order of magnitude better than previously reported interferometric methods. This great sensitivity over the whole 1100-1700 $\mathrm{nm}$ spectral range, together with the simplicity and ease of manipulation, brings back the interferometric method as a powerful tool for detailed investigation of dispersion properties of optical fibers.

\section{ACKNOWLEDGMENT}

The authors wish to thank Prof. G. Guekos from the Swiss Federal Institute of Technology, Zurich, for valuable comments and $\mathrm{V}$. de Coulon for helpful measurements.

\section{REFERENCES}

[1] L. G. Cohen and C. Lin, "A universal fiber-optic measurement system based on a near-IR fiber Raman laser," IEEE J. Quantum Electron., vol. QE-14, pp. 855-859, 1978.

[2] B. Costa, M. Puleo, and E. Vezzoni, "Phase-shift technique for the measurement of chromatic dispersion in single-mode optical fibers using LED's," Electron Lett., vol. 19, pp. 1074-1076, 1983.

[3] -M. Tateda, N. Shibata, and S. Seikai, "Interferometric method for chromatic dispersion measurement in a single-mode optical fiber," IEEE J. Quantum Electron., vol. QE-17, pp. 404-407, 1981. 
[4] H. T. Shang, "Chromatic dispersion measurement by white-light interferometry on meter-length single-mode optical fibers," Electron. Lett., vol. 17, pp. 603-605, 1981.

[5] L. G. Cohen and J. Stone, "Interferometric measurements of minimum dispersion spectra in short lengths of single-mode fiber," Electron. Lett., vol. 18, pp. 564-566, 1982.

[6] J. Stone and L. G. Cohen, "Minimum-dispersion spectra of singlemode fibers measured with subpicosecond resolution by white-light crosscorrelation," Electron. Lett., vol. 18, pp. 716-718 1982.

[7] L. G. Cohen, "Comparison of single-mode fiber dispersion measurement techniques," J. Lightwave Technol., vol. LT-3, pp. 958-966, 1985 .

[8] W. D. Bomberger and J. J. Burke, "Interferometric measurement of dispersion of a single-mode optical fiber," Electron Lett., vol. 17, pp. 495-496, 1981

[9] F. Mengel, "Interferometric monomode fiber measurement: Influence of source spectrum and second-order dispersion," Electron. Lett., vol. 20, pp. 66-67, 1984.

[10] J. F. Nye, Physical Properties in Crystals. Oxford: Clarendon, 1960

[11] D. Gray, Ed., American Institute of Physics Handbook. New York: McGraw Hill, 1976.

[12] R. Ulrich and A. Simon, "Polarization optics of twisted single-mode fibers," Appl. Opt., vol. 18, pp. 2241-2251, 1979.

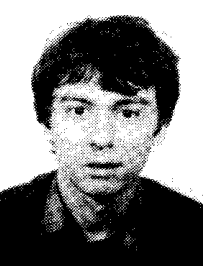

Luc Thévenaz received the B.Sc. degree in astrophysics from the Observatory of Geneva, Switzerland, in 1982, and the M.Sc. degree in physics from the University of Geneva in 1986.

In 1983, he joined the Group of Applied Physics, University of Geneva, Switzerland, where he has been engaged in research work on single-mode fibers measurement techniques. His current interests are in interferometric methods for chromatic dispersion and birefringence measurements and in new refractive index profile design of single-mode fibers.

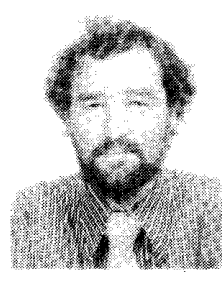

Jean-Paul Pellaux received the Ph.D. degree in physics from the University of Neuchâtel, Switzerland, in 1978 .

He joined the Microtechnique Institute of University in Neuchâtel working on holographic coupling of optical fibers. Later he was engaged in heading the Research and Development Staff in Cabloptic Fiber Manufacture. In 1982 he joined the Applied Physics Group, University of Geneva, Switzerland. He is now Manager of the Development Group in Alphatronix.

Jean-Pierre Von der Weid received the $\mathrm{Ph} . \mathrm{D}$. degree in physics from Pontificia Universidade Catholica (PUC), Rio De Janeiro, Brazil, in 1975. He joined the Physics Institute, University of Neuchâtel, Switzerland, working on solid-state physics. He then returned as Professor to the Physics Department of PUC University, Rio de Janeiro, Brazil. He spent one year in 1986 in the Applied Physics Group, University of Geneva, Switzerland, where he investigated chromatic dispersion and birefringence measurement techniques. 